\title{
CaM Kinase I Regulation of p53 in Breast Cancer Cells
}

\author{
Renee C. Geck, Cody Coblentz, Angela Rofelty and John M. Schmitt* \\ The Biology Department, George Fox University, 414 N. Meridian St, Newberg, OR 97132, United States \\ Email: jschmitt@georgefox.edu
}

\begin{abstract}
Ca}^{2+} /$ calmodulin-dependent protein kinases (CaM Kinases) are intracellular signaling targets of hormones implicated in numerous cancers including breast cancer. The hormone estrogen (E2) or the muscarinic receptor agonist carbachol activate CaM Kinases, ERK and transcription factors to promote cell growth and differentiation. The mechanism by which E2 activates CaM Kinases and their precise intracellular targets that control cancer cell growth and survival remain to be elucidated. We show that E2 treatment of MCF-7 cells activates a CaM KI, ERK and cell growth pathway through the estrogen receptor alpha (ER $\alpha)$. Treatment of MDA-MB-231 (MDA) cells with carbachol also stimulated this pathway. Interestingly, we identified a CaM KI phosphorylation site within the C-terminal region of the tumor suppressor, p53, at serine 366 (S366). Stimulation of cells using either E2 or carbachol triggered phosphorylation of p53 at S366, which was blocked by pretreatment with the CaM KK inhibitor, STO-609 or transfection with siRNAs directed against CaM KI. Cells transfected with constitutively-active CaM KK revealed an elevated level of endogenous p53 phosphorylation at S366. Purified p53 associated with CaM KI from MCF-7 and MDA lysates and immunoprecipitation of transfected Flag-CaM KI revealed the presence of endogenous p53. Evaluation of agonist-stimulated cells using confocal microscopy suggests that endogenous CaM KI and p53 proteins co-localize in the cytoplasm. The p53 inhibitor, Pifithrin-alpha (PFT- $\alpha$ ) increased p53 cytoplasmic localization, cell growth and survival. Treatment of MCF-7 or MDA cells with E2 or carbachol promoted cell growth and that was blocked by STO-609 but not PFT- $\alpha$. This data is the first to suggest CaM KI may phosphorylate and regulate p53 in breast cancer cell growth and survival.
\end{abstract}

Keywords: Estrogen (E2), Carbachol, Calcium/calmodulin-dependent protein kinase kinase (CaM KK), Calcium/calmodulin-dependent protein kinase I (CaM KI), Extracellular-Regulated Protein Kinase (ERK), p53

\section{Introduction}

Calcium $\left(\mathrm{Ca}^{2+}\right)$ is a second messenger in cellular signaling pathways that controls transcription, cell cycle, and apoptosis, and thus contributes to tumor growth and development [1]. Some of these signals are sensed by $\mathrm{Ca}^{2+}$ binding the protein calmodulin $(\mathrm{CaM})$, which then binds a wide variety of target proteins [2]. The multifunctional $\mathrm{Ca}^{2+} /$ calmodulin-dependent protein kinase (CaM K) family of serine/threonine kinases includes CaM K kinase (CaM KK), CaM KII, and closely related CaM KI and CaM KIV [3]. CaM Ks are activated by CaM binding, and phosphorylate many substrates due to the low specificity of their target sequences $[1,3,4]$. CaM KK's target proteins include CaM KI, CaM KIV, AMPK, and Akt, through which CaM KK regulates numerous processes including proliferation, survival, stemness, cell morphology, glucose and lipid metabolism, and immune cell function $[1,4]$. CaM KK phosphorylation of CaM KI relieves autoinhibition and promotes CaM KI-mediated processes including neural plasticity, cell cycle control, proliferation, and migration and invasion of tumor cells $[1,4]$. CaM KI and CaM KIV phosphorylate similar target sequences, but differ in their intracellular localization: CaM KI is cytosolic or membrane anchored, whereas CaM KIV is primarily nuclear [3]. CaM KII is most highly expressed in neurons and does not require phosphorylation by CaM KK, as it autophosphorylates and functions as a multimer to control neurotransmitter synthesis and synaptic plasticity $[1,3]$.

Key downstream targets of CaM Kinase signaling for cell cycle regulation include mitogen-activated protein kinase (MAPK) and cyclin-dependent kinase (CDK) families. $\mathrm{Ca}^{2+}$ leads to extracellular-signal- 
regulated kinase (ERK) and c-Jun N-terminal kinase (JNK) activation through CaM KK and CaM KI in neurons and neuroblastoma cells [5,6], and estrogen (E2) promotes ERK activation in MCF-7 breast cancer cells dependent on CaM KK and CaM KI[7]. CaM Kinases also promote CDK activity, such that CaM K family inhibition or expression of kinase-dead CaM KI arrests fibroblasts in G1 due to loss of CDK4 and cyclin D1 activity [8]. Treatment of MCF-7 cells with E2 or the M3 muscarinic receptor agonist, carbachol, increases cyclin D1 levels through CaM KK and ERK [7], confirming that this pathway occurs in multiple cell types, though it is not universal [8]. Though the complete mechanisms remain to be determined, CaM KI does not directly phosphorylate CDK4 or cyclin D1 since they are primarily nuclear [8]. Instead, CaM KI alters cell cycle gene expression through promoting activity of CREB binding proteins [9, 10], and by phosphorylating p27 to stabilize CDK4/cyclin D1 complexes [11]. Greater understanding of CaM Kinase regulation of the cell cycle is necessary to determine mechanisms of control in diverse cell types and physiological settings.

Due to their roles in growth, proliferation, and cell morphology, many CaM Kinases are overexpressed or activated in tumors [1]. CaM KK expression is increased in hepatocellular carcinoma (HCC), glioma, and acute myeloid leukemia (AML), where it correlates with poor prognosis, and is also elevated in prostate and ovarian tumors compared to adjacent normal tissue [1, 12]. CaM KI is overexpressed in many cancer types, including breast cancer and medulloblastoma (MB) [1], and can promote migration of MB cells [13]. CaM Kinases can also promote tumor formation though alterations of cellular metabolism; CaM KK promotes lipid accumulation in hepatocytes and increases formation of prostate tumors in mouse models through increased lipid synthesis [14, 15].

Their roles in tumor formation make the CaM Kinases potential targets for anti-tumor therapies. Specific inhibition of CaM KK by the ATP-competitive inhibitor STO-609 decreases proliferation of AML, prostate, gastric, HCC, and ovarian cancer cells, and decreases tumor growth in mouse models of prostate cancer and HCC $[1,16]$. In MCF-7 cells, inhibiting or depleting CaM KK or CaM KI in leads to G1 arrest due to reduction of cyclin D1 and phospho-Rb [17], and cell growth resulting from treatment with E2 or carbachol is blocked by STO-609 [7]. In spite of the growing role for CaM Kinases in health and disease, determining their upstream activators, such as receptors, and downstream targets remain to elucidated in specific disease settings.

Approximately $70 \%$ of breast cancers express estrogen or progesterone receptors (ER and PR), and generally have better prognosis than hormone receptor-negative tumors [18, 19]. Breast tumors reliant on ER for proliferation and survival can be treated with the E2 antagonist tamoxifen [19]. ER can respond to E2 through the genomic pathway, where ER translocates to the nucleus and acts as a transcription factor, or the non-genomic pathway where ER at the membrane or cytosol initiates signaling cascades [19]. The requirement of $\mathrm{Ca}^{2+}$ for E2 activation of ERK was the first link between ER and a well-characterized signaling pathway through a non-genomic mechanism: the increase in MAPK signaling in response to E2 is preceded by an increase in intracellular $\mathrm{Ca}^{2+}$, and blocked by $\mathrm{Ca}^{2+}$ chelators [20]. Development of a mouse model that expressed only membrane-localized ER showed nuclear ER is not necessary for E2 activation of ERK in vivo [21]. These studies suggest that ER may utilize intracellular $\mathrm{Ca}^{2+}$ as a signaling molecule to regulate downstream targets including CaM Kinases, although the ER subtype involved in this process remains to be elucidated.

In addition to the pivotal role ERs play in breast cancer development, the tumor suppressor p53 is mutated in $50 \%$ of tumors, including $30 \%$ of breast tumors [22]. P53 is a transcription factor that represses or activates its target genes, which have roles in cell cycle, growth, metabolism, proliferation, and survival [23, 24]. P53 promotes both cell cycle arrest and apoptosis, so tuning this cell fate decision is important when considering therapeutic strategies [24]. Over 150 post-translational modifications, including phosphorylation events, have been identified on p53, and regulate p53 levels, activity, and interactions with other proteins [23]. Phosphorylation of S15 and T18 activate and stabilize p53, and S46 phosphorylation specifically promotes pro-apoptotic activity of p53 [23, 25]. Other sites are inhibitory, such as S315 and S376, which are phosphorylated by GSK-3 $\beta$ to increase cytosolic localization of p53, where it is not stabilized and cannot induce apoptosis [26]. The c-terminal regulatory region of p53 contains many additional phosphorylation sites that tune its stability, subcellular localization, and activity [23, 27]. Many of these sites can be phosphorylated by multiple kinases, likely because redundancy is a fail-safe mechanism for these important regulatory events [27, 28].

Here, we show that activation of ER $\alpha$ leads to increased growth and survival as well as CaM KI activation of ERK in breast cancer cells. We also identify a novel interaction between CaM KI and p53 
in the cytoplasm, and show that CaM KI phosphorylates p53 on S366 in response to agonists that elevate intracellular calcium levels. This interaction and phosphorylation may contribute to CaM K signaling promoting growth, survival, and cell cycling in breast cancer cells.

\section{Experimental Procedures}

\subsection{Materials}

The following reagents were purchased from Sigma-Aldrich; 17ß-estradiol (Estrogen; E2), carbachol (carb), Flag (M2) antibody and Flag-agarose conjugated beads, and epidermal growth factor (EGF). Pifithrin-alpha (PFT- $\alpha$ ), protein A/G plus beads, and antibodies that bind to phosphorylated ERK1/2 at Tyrosine 204 (pERK), total ERK1/2 (ERK), $\beta$-Actin, phosphorylated CaM KI at Threonine 177 (pCaM KI), total CaM KI (CaM KI), total p53, cyclin D1, total CaM KII (CaM KII), $\beta$-actin, 14-3-3 $\gamma$, and estrogen receptor alpha $(\mathrm{ER} \alpha)$ were all purchased from Santa Cruz Biotechnology. Scrambled control siRNAs and siRNAs directed against ER $\alpha$ and CaM KI were purchased from Santa Cruz Biotechnology. Antibodies that bind to phospho-CaM KII at Threonine 286 (pCaM KII) were purchased from Cell Signaling Tech. Inc. Antibodies that bind to phospho-p53 at Serine366 (pS366-p53) were from Abcam and SAB Signaling Antibody. STO-609, MPP, PHTPP (PHT) and FERb were purchased from Tocris Bioscience. U0126 and purified p53 were purchased from EMD MilliporeSigma (USA). Bradford reagent, PVDF and SDS-PAGE reagents were purchased from Bio-Rad. Fluorescent secondary antibodies that were used for western blot imaging were purchased from Li-Cor Biosciences. The transfection reagent, Lipofectamine 2000, fluorescently-conjugated secondary antibodies for confocal microscopy, Tali Dead Cell Red, Tali Cell Cycle reagent, Hoechst 33342 DNA stain, GST antibody, and protein fractionation kits were purchased from Life Technologies (ThermoFisher Scientific).

\subsection{Cell Culture}

The MCF-7 and MDA-MB-231 (MDA) cells and cell culture reagents were purchased from ATCC and cultured at $37^{\circ} \mathrm{C}$, according ATCC guidelines. $70-80 \%$ confluent MCF-7 or MDA cells were placed in serum-free base media overnight, and then stimulated with the designated compounds for western blotting, immunoprecipitation, confocal microscopy, or cell growth assays as indicated. Serum-starved

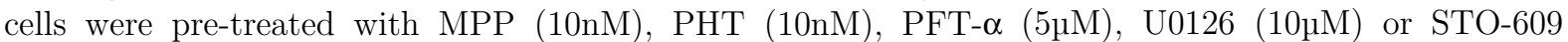
$(5 \mu \mathrm{M})$ for 30 minutes prior to stimulation. MCF-7 or MDA cells were stimulated with E2 (10nM), carb (100mM), FERb (10nM), or EGF (300ng/ml) for 5 minutes or 96 hours as indicated.

\subsection{Transfections}

Equivalent numbers of MCF-7 or MDA cells were plated and grown to 50-60\% confluency and then transfected with the indicated reagent using Lipofectamine 2000. The transfection reagents were prepared according to the manufacturer's guidelines using serum-free base media for 6 hours at $37^{\circ} \mathrm{C}$. Transfection media was then removed and replaced with complete media and the cells were allowed to recover 24 hours prior to serum-starvation and stimulation. siRNA transfections were carried out according to the manufacturer's guidelines and each plate received $20 \mathrm{mM}$ scrambled control siRNA or siRNA directed against ER $\alpha$ or CaM KI (Santa Cruz Biotechnology). Transfection of Flag-CaM KI isoforms (a generous gift from Monika Davare and Tom Soderling at OHSU), control plasmids, and constitutively active CaM KK $\alpha$ (a generous gift from Monika Davare and Tom Soderling at OHSU), were performed at $2.5 \mu \mathrm{g}$ per plate using Lipofectamine.

\subsection{Western Blotting and Immunoprecipitation}

MDA or MCF-7 cells were transfected, serum-starved and then stimulated as indicated similar to previous studies. The media was aspirated and the cells were lysed using ice-cold lysis buffer consisting of $10 \%$ glycerol, $1 \% \mathrm{NP}-40,50 \mathrm{mM}$ Tris- $\mathrm{HCl}$ at $\mathrm{pH} 7.4,200 \mathrm{mM} \mathrm{NaCl}, 2 \mathrm{mM} \mathrm{MgCl}$. along with the inhibitors PMSF $(1 \mathrm{mM})$, Aprotonin $(2 \mu \mathrm{g} / \mathrm{ml})$, Leupeptin $(1 \mu \mathrm{g} / \mathrm{ml})$ and Sodium Orthovanadate $(1 \mathrm{mM})$. 
Ice-cold whole cell extracts were spun for 5 minutes at $4^{\circ} \mathrm{C}$ to pellet nucleotides and cellular debris. Exactly $5 \mu \mathrm{M}$ of supernatant from each treatment was then aliquoted into the wells of a 96-well plate and quantified using the Bradford protein assay and reagent (Bio-Rad). Bovine serum albumin standards and samples were then read using a Bio-Rad 680 micro-plate reader. Equivalent protein amounts were then used for either immunoprecipitation or western blotting. Briefly, $500 \mu \mathrm{g}$ of each lysate was used and their volumes normalized in ice-cold lysis buffer for immunoprecipitation and incubated overnight at $4^{\circ} \mathrm{C}$ with their respective primary antibodies and agarose. Precipitates were spun at $4^{\circ} \mathrm{C}$ and washed $2 \mathrm{x}$ in ice-cold lysis buffer, run on SDS-PAGE gels and analyzed by western blotting. Lysates or precipitates were separated by SDS-PAGE, blotted onto PVDF membranes using a Bio-Rad Turbo Transfer system and blocked in TBS with $5 \%$ milk for 1 hour at room temperature. PVDF membranes were probed overnight at $4^{\circ} \mathrm{C}$ using the indicated primary antibodies in TBS Tween $(0.1 \%)$ with $5 \%$ Bovine serum albumin, washed $4 \mathrm{x}$, and probed with the appropriate fluorescent-conjugated secondary antibody in TBS Tween (0.1\%). PVDF membranes were then placed on a black imaging tray and imaged, scanned, densitized and quantified using a Li-Cor Biosciences Odyssey Fc. Band intensities were normalized to loading control lysates and to the untreated control lanes and presented as fold phosphorylation, binding (association), or protein expression as appropriate.

\subsection{Cell Fractionation}

MCF-7 cells were fractionated using the ThermoScientific cultured cell kit and protocol. Equivalent numbers of MCF-7 cells were serum-starved overnight and stimulated as indicated with E2 or PFT- $\alpha$. Cells were trypsinized, pelleted and washed with ice-cold PBS. Equal numbers of cells were transferred to microcentrifuge tubes, pelleted, and incubated with ice-cold CEB buffer. Cell lysates were then centrifuged and incubated with MEB and NEB buffers to separate the cytoplasmic, membrane and nuclear extract components. Cytoplasmic and nuclear extracts were then examined using the Bradford assay and analyzed by western blotting with the indicated primary antibodies.

\subsection{Confocal Microscopy}

Equivalent numbers of MDA and MCF-7 cells were plated and cultured on glass coverslips in 6-well plates according to ATCC guidelines. Cells were serum-starved, pretreated and then stimulated with E2 or carb as indicated for 5 minutes. Immediately following treatment, culture media was aspirated from the cells and the cells washed once with PBS and then fixed with a $4 \%$ paraformaldehyde, $4 \%$ sucrose, $50 \mathrm{mM}$ HEPES in PBS at pH 7.5 for 15 minutes at room temperature. Cells were gently washed three times using PBS with $3 \%$ bovine serum albumin (BSA) for 5 minutes each. PBS was aspirated and the cells were permeabilized with $0.2 \%$ Triton X-100 in PBS for 15 minutes at room temperature. Cells were washed twice with PBS containing 3\% BSA (PBS-BSA) which was aspirated and replaced with PBSBSA containing the indicated primary antibody at a 1:100 dilution for 60 minutes at $37^{\circ} \mathrm{C}$. Primary antibodies were aspirated, cells washed twice with PBS-BSA, and then incubated with PBS-BSA containing fluorescently-conjugated secondary antibodies for 60 minutes at room temperature. Secondary antibodies were aspirated, cells were washed twice with PBS-BSA and incubated with Hoechst stain (1:30,000) for 15 minutes at room temperature. Finally, the stain was aspirated, cells washed three times with PBS for 5 minutes each and coverslips mounted on glass slides for confocal imaging. Cells were imaged and analyzed with the 63X objective using a Leica SPE TCSII inverted confocal microscope and LAS X software.

\subsection{Cell Cycle Assay}

MCF-7 cells were analyzed to determine the appropriate phase of the cell cycle that they exhibited using the Tali Cell Cycle Kit (Invitrogen, ThermoFisher). MCF-7 cells were plated at equal numbers and grown to 50-60\% confluency, serum-starved and pre-treated with PFT- $\alpha$ and then stimulated with E2, E2 alone or PFT- $\alpha$ for 96 hours as indicated. Cells were then trypsinized and pelleted by centrifugation at $500 \mathrm{X} \mathrm{g}$ for 5 minutes followed by their resuspension in equal volumes of Dulbecco's Phosphate Buffered Saline (DPBS). The recommended concentration of cells was placed into individual microcentrifuge tubes, centrifuged at $500 \mathrm{Xg}$ for 5 minutes, then the supernatant was aspirated and the 
cells were gently resuspended with ice-cold $70 \%$ ethanol and placed on ice. Cells were allowed to fix overnight at $-20^{\circ} \mathrm{C}$, centrifuged at $1000 \mathrm{X}$ g for 5 minutes at $4^{\circ} \mathrm{C}$ and resuspended in $1 \mathrm{ml}$ of DPBS and then centrifuged again for 10 minutes at $4^{\circ} \mathrm{C}$ to remove the DPBS. Cells were resuspended in $200 \mu \mathrm{L}$ of Tali Cell Cycle Solution using the recommend cell concentration and incubated in the dark at room temperature for 30 minutes. Finally, cells were gently resuspended and loaded into Tali analysis slides and read using the Tali Image-based cytometer with the Cell Cycle software. As recommended by the manufacturer, the control untreated sample cells were used to set the appropriate cell size gate and to exclude any cellular debris or aggregates for final sample analysis.

\subsection{Cell Growth and Apoptosis Assay}

MCF-7 and MDA cells were analyzed for live cell viability and dead cell with the Tali Viability Kit Dead Cell Red, according to the manufacturer kit and guidelines (Invitrogen, ThermoFisher). Equal numbers of MCF-7 or MDA cells were plated and grown to 50-60\% confluency, serum-starved and then treated with the indicated inhibitors and agonists for 96 hours. Briefly, treated cells were trypsinized, centrifuged and resuspended with Dulbecco's Phosphate Buffered Saline (DPBS) at the recommended cell concentration. $100 \mu \mathrm{L}$ of cells from each treatment were aliquoted into fresh microcentrifuge tubes and incubated with $1 \mu$ l of Tali Dead Cell Red at room temperature in the dark for 5 minutes. Next, 25 $\mu \mathrm{L}$ from each treatment containing the Dead Cell Red was loaded into a Tali analysis slide and immediately read using the Tali Image-based cytometer to measure the number of live and dead cells.

\subsection{Statistics}

To determine if statistically significant differences existed between specific experimental groups, Student $t$-tests were performed on the data. Significance levels were set at $0.05(95 \%)$ or higher and significant $P$ values are indicated in all figures as either "*" which indicates $P \leqslant 0.05$ or "**" which indicates $P \leqslant$ 0.01 .

\section{Results}

\subsection{ER $\alpha$ Activation Stimulates Cell Growth and CaM KI-ERK Signaling}

The ability of compounds and agonists such as ionomycin, carbachol, or E2 to activate CaM Ks in various cells including breast, ovary, and prostate types have previously been established [29-31]. E2 is capable of activating ER subtypes that may lead to genomic and rapid non-genomic signaling and cell phenotypes. To investigate which ER receptors are responsible for promoting MCF-7 cell growth, breast cancer cells were pretreated with the ER $\alpha$ or ER $\beta$ selective antagonists followed by stimulation with a physiologically relevant concentration of E2. The ER $\alpha$ antagonist, MPP, significantly blocked the ability of E2 to promote cell growth, whereas the ER $\beta$ antagonist PHTPP (PHT) had no effect on E2stimulated growth (Fig. 1A). The ER $\beta$ agonist, FERb, did not increase cell growth as compared to the control (Fig. 1A).

Since E2 treatment of MCF-7 cells increased cell growth and has previously been shown to activate ERK through CaM Ks [7] we next examined the ability of MPP to block E2 activation of ERK. E2 treatment of MCF-7 cells increased the phosphorylation and activation of endogenous ERK1/2 within 5 minutes and was blocked by pre-treating cells with MPP (Fig. 1B). Similarly, CaM KI activation and phosphorylation at threonine 177 was increased by E2 and inhibited by MPP (Fig. 1B). To precisely evaluate the ER subtype required for CaM KI activation of ERK, MCF-7 cells were transfected with control scrambled siRNAs (siControl) or siRNAs directed specifically against ER $\alpha(\operatorname{siER} \alpha)$. A 5-minute stimulation of siControl cells with E2 increased ERK activation as measured by phosphorylation at tyrosine 204, whereas siER $\alpha$ completely blocked E2 activation of ERK (Fig. 1C). To confirm the specificity and gene silencing effect of the siRNAs, transfected MCF-7 cell lysates were evaluated for the

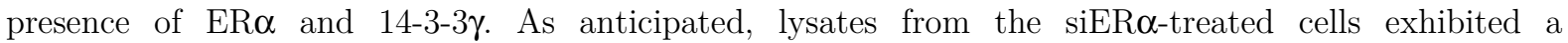
significantly reduced expression of endogenous ER $\alpha$ but not 14-3-3 $\gamma$ proteins (Fig. 1D). To further evaluate the receptor selectivity for the siRNA transfections, cells were transfected with siControl or 
siER $\alpha$ and then treated with epidermal growth factor (EGF) for 5 minutes, which activates ERK independently of ER $\alpha$ [32]. EGF treatment of MCF-7 cells increased ERK activation in the presence of control siRNA or siER $\alpha$ as expected. Taken together, these data suggest that E2 is capable of activating CaM KI, ERK and cell growth specifically through ER $\alpha$.

To establish the ability of E2 to rapidly activate CaM KK and CaM KI, MCF-7 cells were pretreated with the selective inhibitor of CaM KK, STO-609 (STO) and then stimulated with E2. Cells treated with E2 for 5 minutes exhibited an increased phosphorylation and activation of CaM KI that was completely blocked by inhibiting CaM KK (Fig. 2A). To expand the ability of additional calcium signaling compounds and cells that may utilize CaM Kinase signaling for cell growth, we treated the MDA-MB-231 triple negative breast cancer cells (MDA) with the muscarinic receptor agonist carbachol (Carb). Previous studies have shown that carbachol can activate CaM Ks and ERK; therefore, MDAs were pretreated with either the CaM KK inhibitor STO-609 or the MEK and ERK inhibitor, U0126 (U0) before treatment with carbachol for 5 minutes (Fig. 2B). Carbachol activates both endogenous CaM KI and ERK in MDAs, both of which are inhibited by pretreatment with STO-609. CaM KI activity is not blocked by the MEK inhibitor U0126, but ERK activation is significantly blocked (Fig. 2B). These observations suggest that ERK activation is downstream of CaM KK and CaM KI signaling in MDAs.

CaM KII is related to CaM KI, activated by calcium, and has been identified in several types of cancer cells including MDAs [33]. To examine the possible role for CaM KII in our system as well as control for the specificity of STO-609 and U0126, MDAs were pretreated with STO or U0 (Fig. 2C). Next, MDAs were stimulated with carbachol and endogenous CaM KII activation and phosphorylation at threonine 286 were examined by western blot. Carbachol activated CaM KII in MDAs, but neither inhibition of CaM KK (STO) nor ERK (U0) had an effect on CaM KII activity (Fig. 2C). Taken together, these data suggest that E2 and carbachol are sufficient to activate ERK through CaM KK and CaM KI in breast cancer cells, while CaM KII is activated independent of CaM KK or ERK.

\subsection{E2 Promotes CaM KI Phosphorylation of p53}

Given the ability of E2 and carbachol to promote cell growth and activation of CaM Ks, as well as previous studies linking them to control of cell cycle proteins and p27 [34, 35], we hypothesized that CaM Ks could crosstalk with other cell cycle proteins such as p53. P53 is a key regulator of cell growth and survival, frequently mutated in cancer, and has numerous regulatory phosphorylation sites [27]. The number of reported substrates for CaM KI is limited; however evaluation of the amino acid sequence of p53 revealed a preferred CaM KI phosphorylation site at S366, within the c-terminal regulatory region of p53 (Fig. 2D) [27]. To evaluate whether p53 was phosphorylated at S366 in our system, MCF-7 cells were stimulated with E2, and endogenous p53 phosphorylation at S366 (pS366-p53) was assayed by western blotting. Interestingly, E2 promoted a strong increase of pS366-p53 that was not blocked by the ERK inhibitor, U0126, but was significantly inhibited by blocking CaM KI activity with STO-609 (Fig. $2 \mathrm{E})$. Since carbachol was sufficient to activate CaM KI in MDAs, we also investigated endogenous p53 phosphorylation by pretreating MDAs with STO-609. Carbachol stimulation of MDAs promoted a twofold increase in pS366-p53, which was significantly inhibited by blocking CaM KI activation (Fig. 2F). These data suggest that E2 and carbachol promote p53 phosphorylation dependent on CaM KI activity.

To investigate the possible requirement of CaM KI in phosphorylating p53 at S366, we transfected MCF-7 cells with siControl or siRNA directed against CaM KI (siKI). E2 treatment of siControltransfected cells significantly increased pS366-p53 within 5 minutes, whereas this increase in pS366-p53 was significantly blocked in cells expressing siKI (Fig. 3A). To confirm on-target effects of siKI, western blot analysis of MCF-7 cells transfected with either siControl or siKI revealed a significant reduction of endogenous CaM KI protein expression, while endogenous ERK protein levels were unaffected (Fig. 3B).

We also evaluated whether $\mathrm{CaM} \mathrm{KK}$ and $\mathrm{CaM} \mathrm{KI}$ were sufficient to promote S366-p53 phosphorylation. Transfection of MCF-7 cells with plasmids containing constitutively active CaM KK (c.a. KK) but not control pcDNA3 plasmids (C) significantly enhanced endogenous pS366-p53 but not total p53 levels (Fig. 3C). We also examined the requirement for the carbachol-CaM K pathway to increase pS366-p53 in MDA cells. MDA cells were transfected with siControl or siKI and then stimulated with carbachol. Carbachol treatment rapidly increased pS366-p53 in siControl cells with no effect on total p53 levels, but this increase in pS366-p53 was significantly blocked by transfection with 
siKI (Fig. 3D). We confirmed that endogenous CaM KI protein levels were decreased by siKI whereas total ERK protein levels were not affected (Fig. 3E). Similar to our observations in MCF-7 cells, overexpression of c.a. KK in MDAs caused a significant increase in pS366-p53 with no effect on endogenous p53 total protein levels (Fig. 3F). Taken together, these observations suggest that E2 and carbachol signal through CaM KI to increase p53 phosphorylation on S366, and that CaM KK activation is sufficient to increase pS366-p53.

\subsection{CaM KI Binds and Co-Localizes with p53 in the Cytosol}

Since previous studies have shown that CaM KI stably binds cell cycle proteins that it phosphorylates [11], we sought to determine if CaM KI associates with p53. As an initial approach, we incubated purified glutathione-S-transferase protein (GST) or GST-tagged p53 with cell lysates from either MCF-7 or MDA cells. Immunoprecipitation of GST from control untreated MCF-7 lysates did not associate with CaM KI protein as analyzed by western blot (Fig. 4A). In contrast, endogenous CaM KI protein precipitated with GST-p53 from MCF-7 cells that had been stimulated with E2 for 5 minutes. Similarly, carbachol treatment of MDA cells also revealed a significant interaction between GST-p53 and CaM KI protein (Fig. 4A).

We also evaluated the ability of the proteins to interact inside of whole cells by transfecting cells with plasmids containing wild-type, Flag-tagged CaM KI, and then treating them with E2 and immunoprecipitating with anti-Flag antibody. Transfected, unstimulated MCF-7 cells (control) showed a basal association of endogenous p53 with Flag-CaM KI, and stimulation with E2 for 5 minutes significantly increased the association of endogenous p53 and CaM KI (Fig. 4B). To control for nonspecific protein binding to overexpressed CaM KI, we also examined the immunoprecipitates for the presence of the cell cycle protein, cyclin D1. Cyclin D1 protein was present in MCF-7 whole cell lysates (WCL) but did not bind to Flag-CaM KI (Fig. 4B). The ability of carbachol to promote Flag-CaM KI and p53 binding was also evaluated in MDA cells. Carbachol treatment significantly increased the association of CaM KI and p53 (Fig. 4C). Consistent with observations in MCF-7 cells, cyclin D1 was not detected in the MDA Flag immunoprecipitates (data not shown). These data suggest that CaM KI associates with p53, and that E2 and carbachol promote this interaction.

To further evaluate the association of CaM KI with p53 under physiological conditions, endogenous proteins from MCF-7 and MDA cells were immunoprecipitated and analyzed by western blotting. MCF7 cells were serum-starved and left untreated or pretreated with the CaM K-inhibitor, STO-609 followed by stimulation with E2 for 5 minutes. Immunoprecipitation of endogenous CaM KI proteins from control, E2-treated, or STO-609 pretreatment followed by E2-stimulation, revealed an association of endogenous p53 (Fig. 5A). The binding of p53 and CaM KI was increased in the presence of E2 and significantly reduced by inhibiting CaM KI activity with STO-609 (Fig. 5A). We also performed reciprocal immunoprecipitations with endogenous p53 from MCF-7 cells lysates followed by western blotting for CaM KI. P53-CaM KI protein association was enhanced with E2-treatment of cells and significantly reduced with STO-609 (Fig. 5B). We also examined the ability of endogenous CaM KI and p53 to associate in MDA cells in the presence and absence of carbachol. Carbachol treatment of MDAs for 5 minutes increased CaM KI binding to p53 in a manner that was dependent on CaM K activity as it was sensitive to STO-609 (Fig. 5C).

We also evaluated CaM KI-p53 protein association within E2-treated breast cancer cells by analyzing immunofluorescently-labelled proteins using confocal microscopy. MCF-7 cells that were treated with E2 for 5 minutes and labelled with fluorescent antibodies to endogenous CaM KI revealed its presence in the cytoplasm and perinuclear region (Fig. 5Di). Endogenous p53 was also observed in the cytoplasm of E2-treated MCF-7 cells (Fig. 5Dii). Examination of both proteins within cells demonstrated that CaM KI and p53 co-localized within the cytoplasm of MCF-7 cells (Fig. 5Diii). The cellular location of endogenous CaM KI and p53 was also examined carbachol-treated MDA cells. Similar to MCF-7 cells, CaM KI (Fig. 5Ei) and p53 (Fig. 5Eii) proteins were both observed within the cytoplasm of stimulated MDAs. The analysis of the overlay of both proteins inside MDA cells revealed that they co-localized within the cytoplasm and perinuclear region (Fig. 5Eiii). The examination of CaM KI-p53 association from cellular lysates, as well as their subcellular location within cells by confocal microscopy, suggests that the proteins may bind within the cytoplasm of breast cancer cells. 
Our data suggest that CaM KI is sufficient to phosphorylate and associate with p53 in the cytoplasm of certain cell types. Therefore, we investigated the subcellular location and phosphorylation state of p53 in E2-treated MCF-7 cells. MCF-7 cells were serum-starved, stimulated with E2, or treated with the compound PFT- $\alpha$, which specifically inhibits p53 activity by preventing its nuclear localization and binding to DNA [36-40]. The cell lysates were fractionated and purified to separate nuclear $(\mathrm{N})$ and cytoplasmic (Cyt) fractions. Western blot analysis of purified fractions from control cells suggests that endogenous p53 was present in both the nucleus and cytoplasm but was not phosphorylated on S366 (Fig. 5F). E2 treatment of MCF-7 cells increased the amount of p53 protein within the cytoplasmic fraction of cells (Fig. 5F, upper panel). Phosphorylation of p53 at S366 was also increased within the cytoplasmic component of E2-stimulated cells relative to controls and the nuclear fraction (Fig. 5F, middle panel). PFT- $\alpha$ had no effect on p53 phosphorylation; however, it appeared to increase the cytoplasmic amount of total p53 as compared to the nuclear fraction (Fig. 5F, upper panel). Taken together, these data suggest that E2 promotes CaM KI phosphorylation of p53 within the cytoplasm of cells and reduces nuclear p53 levels.

\subsection{CaM KI and p53 Coordinate to Regulate Cell Growth and Apoptosis}

Previous studies and data presented here have shown that E2, CaM Kinases, and p53 are capable of independently regulating cell growth [7, 41, 42]. However, the ability of these molecules to work in a coordinated pathway to achieve this effect remains uncharacterized. Therefore, we investigated the ability of CaM Kinases working with p53 to regulate the cell cycle in E2-treated MCF-7 cells. Cells were treated with E2 and DNA content was fluorescently evaluated and quantified to determine the fraction of cells in each phase of the cell cycle. The majority of control untreated cells were in the $\mathrm{G}_{1} / \mathrm{G}_{0}$ and $\mathrm{S}$ phases of the cell cycle (Fig. 6A). In contrast, E2 treatment reduced the number of cells in the $\mathrm{G}_{1} / \mathrm{G}_{0}$ phase with an increase in the $\mathrm{S}$ and $\mathrm{G}_{2} / \mathrm{M}$ phases. Treatment of MCF-7 cells with the p53-inhibitor, PFT- $\alpha$, followed by E2 increased the number of cells in the $S$ phase but not $G_{2} / M$, and PFT- $\alpha$ treatment alone resulted in a similar cell cycle profile to $\mathrm{E} 2$ treatment (Fig. 6A). These data suggest that E2 and CaM Kinases may utilize p53 to regulate the cell cycle, and that direct inhibition of p53 with PFT- $\alpha$ enhances cell cycle activity as anticipated.

To further examine the link between CaM KI and p53, we evaluated their participation in MCF-7 and cell growth and apoptosis after 96-hour treatments. E2 stimulation of MCF-7 cells significantly enhanced cell proliferation above serum-starved controls, whereas pretreatment of MCF-7 cells with STO-609 blocked the E2 effect (Fig. 6B). Interestingly, pretreating cells with PFT- $\alpha$ and then E2 significantly enhanced cell growth above control, E2 alone, and STO-E2 levels (Fig. 6B). Inhibition of p53 with PFT- $\alpha$ increased cell growth above control levels although it was 2 to 3-fold less than E2 and PFT- $\alpha$ combined (Fig. 6B). Evaluation of MCF-7 cellular apoptosis revealed the ability of E2-treatment to significantly reduced cell death, an effect that was partially reversed by inhibiting either CaM KI or p53 with STO-609 and PFT- $\alpha$, respectively (Fig. 6C). Treatment of cells with PFT- $\alpha$ alone also reduced apoptosis as compared to controls although it was more than the combination of E2 and PFT- $\alpha$ (Fig. 6C). The data from the MCF-7 cells suggests that E2 utilizes CaM KI and the inhibition of p53 to regulate cell growth and survival.

We also examined the possible connection between CaM KI and p53 in the regulation of MDA cell growth and apoptosis. Carbachol treatment of MDAs significantly enhanced their growth above controls and STO-609 pretreatment blocked carbachol's effect (Fig. 6D). Similar to our observations in MCF-7 cells, pretreating cells with the p53 inhibitor PFT- $\alpha$ followed by carbachol stimulation significantly increased cell growth above carbachol alone. PFT- $\alpha$ treatment also increased cell growth relative to control (Fig. 6D). Serum starvation of MDA cells increased apoptosis at 96 hours, which was blocked by carbachol stimulation (Fig. 6E). Pretreating cells with the CaM KK inhibitor, STO-609 or PFT- $\alpha$ followed by carbachol treatment were also significantly less than controls (Fig. 6E). Treatment of MDAs with PFT- $\alpha$ alone also significantly enhanced cell survival. Taken together, this data suggests that E2 and carbachol require that activity of CaM KI and the inhibition of p53 to promote cell growth and survival. 


\section{Discussion and Conclusion}

We have shown that E2 activates CaM KI and ERK in MCF-7 cells, and also increases a novel interaction between CaM KI and p53, leading to increased p53 phosphorylation at S366. Similarly, carbachol treatment of MDAs promotes CaM KI association with p53 and S366 phosphorylation. CaM KI and p53 associate in the cytoplasm, and this localization is increased with E2 or carbachol and dependent on CaM KK activity. E2 signaling through CaM KI phosphorylation of p53 acts to keeps p53 out of the nucleus. We propose that this prevents p53 from functioning as a tumor suppressor, as it augments the cell cycle progression, cell growth and survival of MCF-7 and MDA cells.

It is known that ER can act through a non-genomic pathway to initiate signaling cascades [20], and we have shown in this and previous publications that E2 activates ERK through a non-genomic pathway involving CaM KK and CaM KI in MCF-7 cells [7, 29]. Our observation that estrogen and carbachol lead to activation of ERK signaling through ER $\alpha$ corroborates reports that the membranelocalized E domain of $\mathrm{ER} \alpha$ is sufficient for E2 activation of ERK in mice [21]. Increases in intracellular calcium have also been measured in MCF-7 cells in response to E2, preceding ERK activation [20], which could activate CaM Ks.

CaM Ks are known to regulate the cell cycle through proteins including cyclin D1, CDK2, CDK4, p21 and p27 [8, 11]. Notably, a study by Mallampalli et al. showed that CaM KI binds and phosphorylates p27, and that the interaction is stable enough that immunoprecipitation of CaM KI also pulls down p27 [11]. This suggests that CaM KI can form complexes with cell cycle proteins, and identification of additional binding partners of CaM KI or other members of these complexes could inform novel signaling mechanisms downstream of CaM Ks. We investigated the interaction between CaM KI and p53 in response to estrogen signaling, but there may be other cell cycle proteins that are also bound and phosphorylated by CaM KI or other CaM Ks in response to various stimuli.

A study by Bailey et al. highlighted interactions between genomic estrogen signaling and p53 [43]. They identified genes in MCF-7 cells that were bound and differentially regulated by ER and p53. ER did not alter p53 levels or displace p53 from these shared genes, but instead independently repressed the expression of the p53 targets. This illustrated a transcriptional mechanism for ER and p53 competition. In this study, we add an additional layer, showing that there are also non-genomic effects of ER that alter p53 and its ability to suppress cell growth and apoptosis.

We specifically identified p53 S366 as a novel phosphorylation target of CaM KI, and showed that pS366-p53 is increased following E2 and carbachol treatment dependent on CaM K signaling. S366 is in the c-terminal regulatory region of $\mathrm{p} 53$, and has been previously characterized in other cell types. However, in many of these settings phosphorylation of S366 leads to activation of p53, which is not what we observed in breast cancer cells. In HEK-293T embryonic kidney cells, phosphomimetic serine to aspartate mutations of S366 and nearby residue S387 increased p53 binding to p21 and AIP promoters, increasing transcription from p53 reporters [44]. Another group also observed that phosphorylation of S366 and other c-terminal residues led to 14-3-3 binding that increased p53 binding to DNA and transcription of its targets [28, 45]. S366 has also been shown to be phosphorylated by CHK1 in response to DNA damage in LNCaP prostate cancer cells [44]. This does not eliminate the possibility that CaM KI can also phosphorylate S366, as many residues on p53 are phosphorylated by multiple kinases [27]. Further investigation of kinase expression and activity in response to different stimuli could help describe different cell types or conditions in which p53 S366 is phosphorylated by specific kinases.

Despite these conflicting results, a study by van Dieck et al. suggested that phosphorylation of S366 can inhibit p53 activity. They observed that phosphorylation of S366 decreases p53 binding to S100A2 by four-fold [46]; p53 activity is increased when it is bound by S100A2 [47]. It would be interesting to measure the binding of S100A2 to p53 in our breast cancer cells, and how it is affected by E2 and carbachol.

Our analysis of cell growth, apoptosis, and the cell cycle profile of breast cancer cells treated with ER agonists and the p53 inhibitor PFT- $\alpha$ further suggest that ER signaling that increases pS366-p53 leads to p53 inhibition. Since PFT- $\alpha$ treatment in addition to E2 or carbachol stimulation has only a small effect on the cell cycle profile and does not significantly increase cell growth, this indicates that most p53 in the cells was already inactivated by signaling downstream of E2 or carbachol and thus could not be further inactivated by PFT- $\alpha$. This aligns with the hypothesis that phosphorylation of S366 by CaM 
KI sequesters p53 in the cytosol. Future studies of phosphomimetic mutants of S366-p53 in breast cancer cells would inform our understanding of the role of this phosphorylation event in this specific setting, since other studies have shown that pS366 can inhibit or activate p53 in different cell types [27, $44,46]$.

Together, our data highlight a new role for non-genomic estrogen signaling in breast cancer cells. Future studies of CaM K targets and binding partners, and of the effects of p53 S366 phosphorylation by CaM Ks, can broaden and deepen our understanding of estrogen and CaM K signaling in breast cancer.

\section{$5 \quad$ Figure Legends}

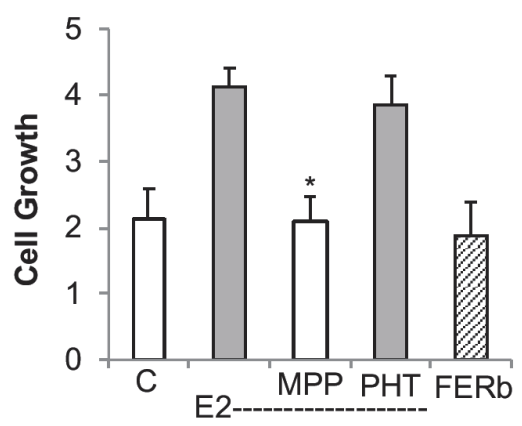

(a)

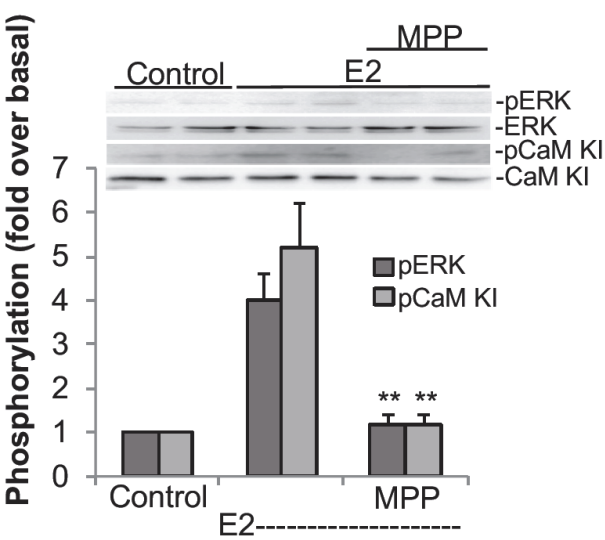

(b)

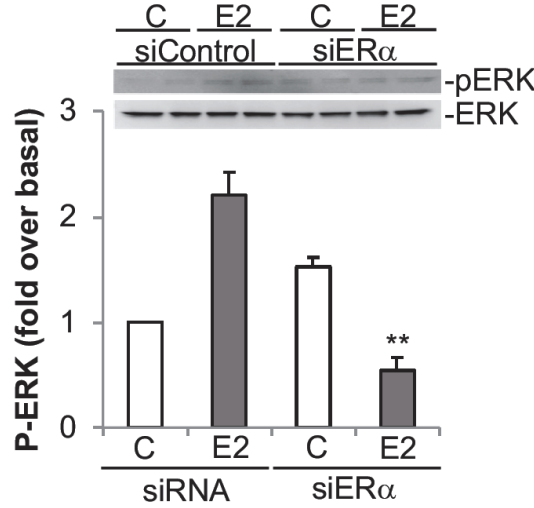

(c)

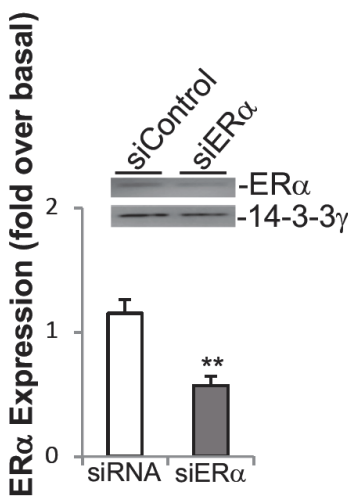

(d)

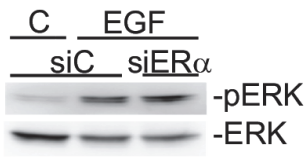

(e)

Fig. 1 Estrogen receptor-alpha mediates activation of CaM KI, ERK and cell growth. a, Estrogen (E2) stimulates MCF-7 cell growth through ER $\alpha$ but not ER $\beta$. MCF-7 cells were treated with FERb (10nM) or E2 $(10 \mathrm{nM})$ for 96 hours in the presence or absence of either the MPP (10nM) or PHTPP (10nM) compounds as described in the "materials and methods" $(\mathrm{N}=4, \pm$ S.E., "** $=\mathrm{p} \leq 0.05)$. The letter "C" represents the control nonstimulated cells in all figures. b, E2 activates CaM KI and ERK through ER $\alpha$. MCF-7 cells were stimulated with E2 for 5 minutes or pretreated with the ER $\alpha$ antagonist, MPP (10nM) as indicated. Endogenous CaM KI and ERK phosphorylation and activation were analyzed and measured by western blotting for phosphorylated-CaM KI (pCaM KI) and phosphorylated-ERK 1/2 (pERK). The lower panels are control western blots from cell extracts visualizing total endogenous ERK1/2 (ERK) and CaM KI protein (CaM KI) used for analysis. Data is presented as fold phosphorylation over control $(\mathrm{N}=6, \pm$ S.E., "**" $=\mathrm{p} \leq 0.01)$. c, Inhibition of ER $\alpha$ blocks ERK activation. MCF-7 cells were transfected with siRNAs and treated as described in the "materials and methods". Cell lysates were analyzed for ERK phosphorylation by western blotting $(\mathrm{N}=6, \pm$ S.E.). d, siRNA directed to ER $\alpha$ blocks receptor expression in MCF-7 cells. Transfected cells were evaluated by western blot for ER $\alpha$ or 14-3-3 $\gamma$ protein expression (N=6, \pm S.E.). e, siRNA to ER $\alpha$ does not inhibit epidermal growth factor (EGF) phosphorylation of 
ERK in MCF-7 cells. MCF-7 cells were transfected with control siRNAs or siRNA targeting ER $\alpha$ and then stimulated with EGF (300ng/ml) for 5 minutes $(\mathrm{N}=3)$.

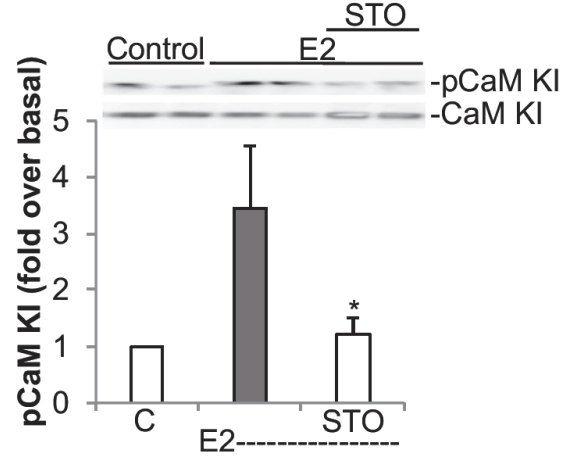

(a)

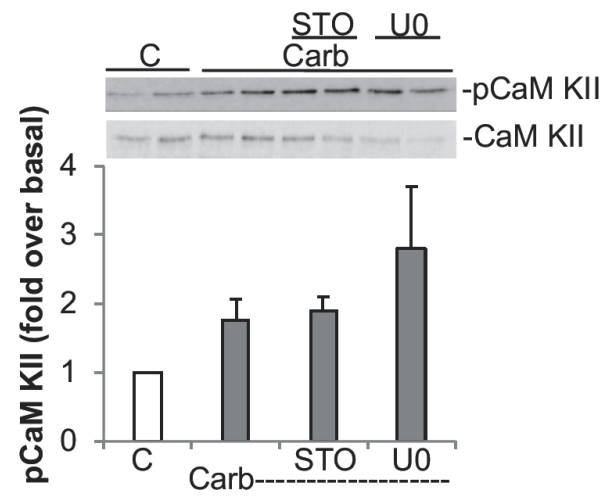

(c)

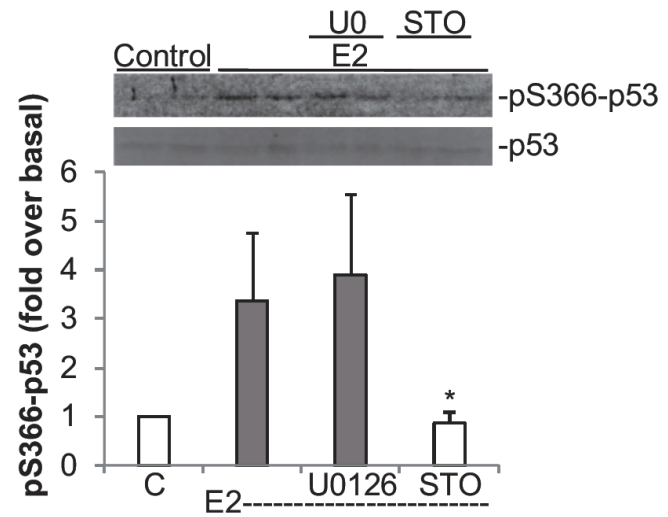

(e)

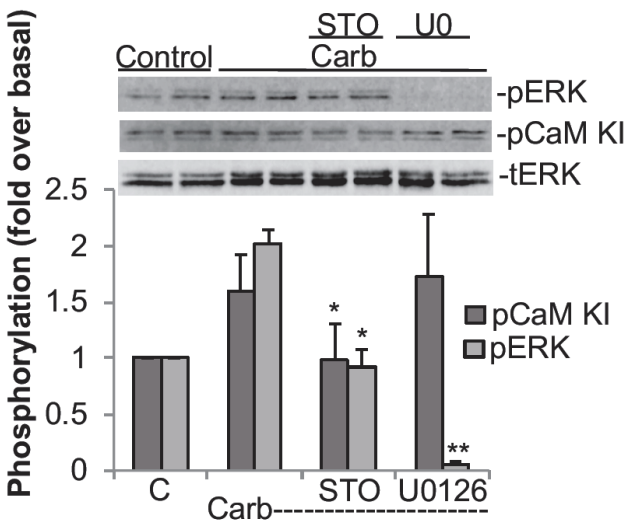

(b)

CaM KI optimal substrate consensus phosphorylation sequence at Serine 366 (underlined, grey S):

$N$ term---------------------

GEYFTLQIRGRERFEMF

RELNEALELKDAQAGK

EPGGSRAHSSHLKSKK

GQSTSRHKKLMFKTEG

PDSD

- C term

(d)

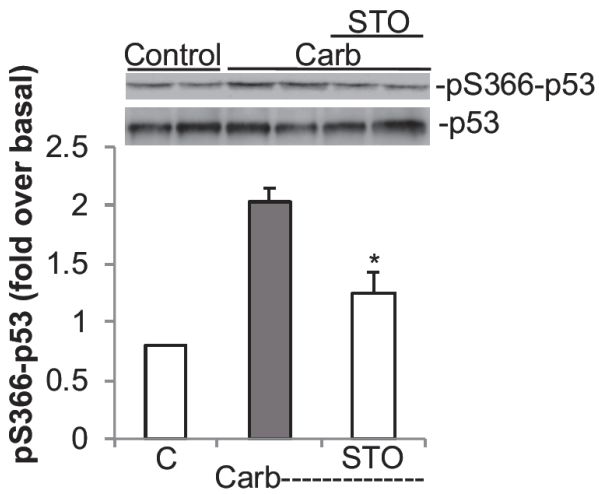

(f)

Fig. 2 E2 and carbachol utilize CaM KK and CaM KI to trigger 53 phosphorylation. a, E2 treatment of MCF-7 cells for 5 minutes induces endogenous activation of CaM KI. MCF-7 cells were pretreated with the selective inhibitor STO-609, then E2 and analyzed by western blot as in figure 1 (N=6, \pm S.E.). b, Carbachol stimulates CaM KK-dependent activation of CaM KI and ERK in MDA-MB-231 (MDA) cells. MDA cells were

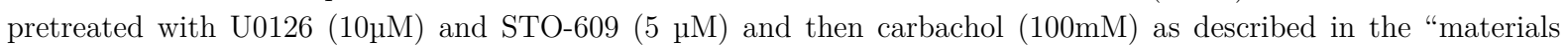
and methods" and protein phosphorylation measured by western blot ( $\mathrm{N}=6, \pm$ S.E.). c, Carbachol activation of CaM KII in MDA cells is STO-609 and U0126 insensitive. MDA cells were treated as in panel b and CaM KII phosphorylation was examined $(\mathrm{N}=6, \pm$ S.E.) d, C-terminal amino acid sequence of p53 and phosphorylation site 
for CaM KI. The optimal CaM KI sequence including serine 366 (S366) is underlined. e, E2 stimulates endogenous p53 phosphorylation at S366 through CaM KK. MCF-7 cells were pretreated similar to panel $\mathbf{b}$ and then stimulated with E2 and p53 phosphorylation at $\mathrm{S} 366$ was analyzed by western blot ( $\mathrm{N}=6$, \pm S.E.). f, Carbachol-dependent phosphorylation of p53 utilizes CaM KK. MDA cells were treated similar to panel $\mathbf{b}$ and p53 phosphorylation was measured and analyzed following a 5 -minute carbachol $(100 \mathrm{mM})$ stimulation $(\mathrm{N}=4, \pm$ S.E.).

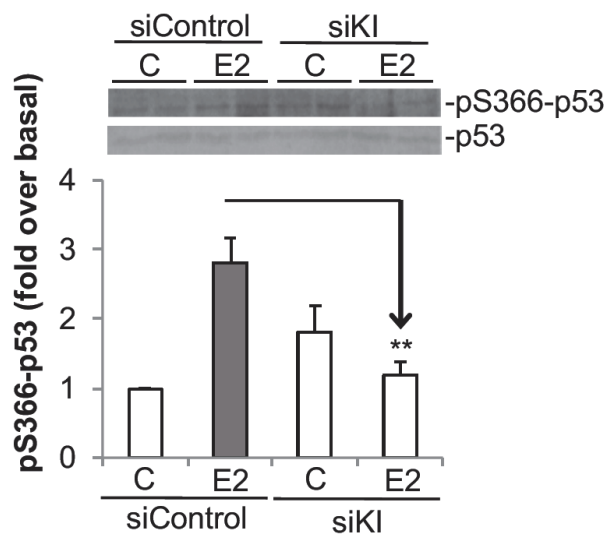

(a)

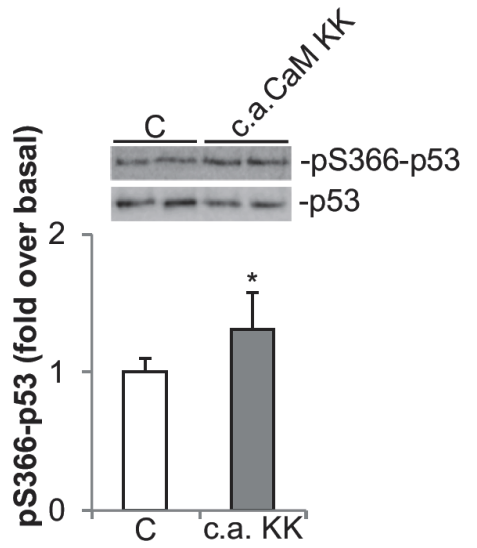

(c)

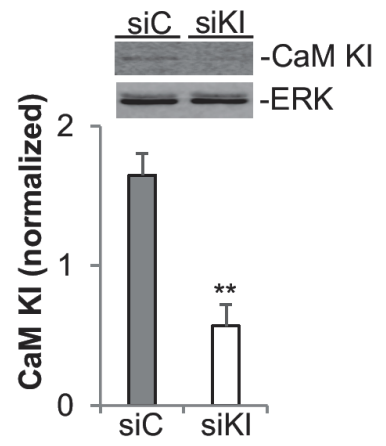

(e)

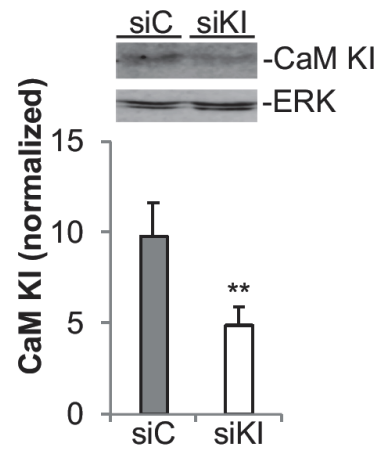

(b)

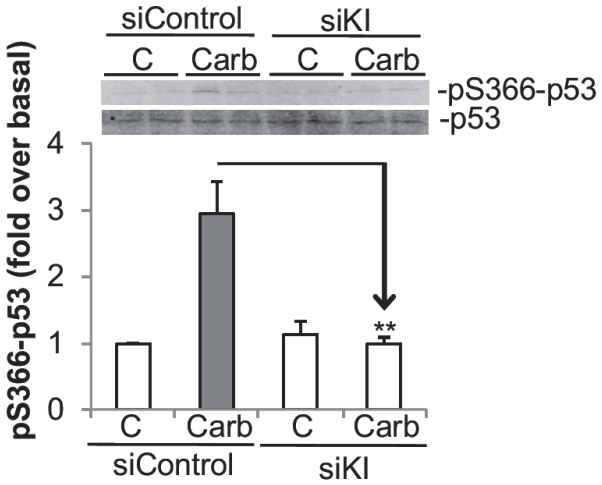

(d)

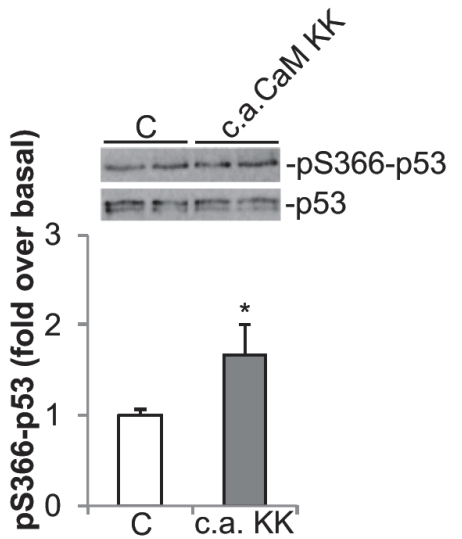

(f)

Fig. 3 CaM KI is required for p53 phosphorylation in breast cancer cells. a, Suppression of CaM KI in MCF-7 cells blocks E2-stimulated phosphorylation of p53 at S366. Cells were transfected with control scrambled siRNAs or siRNA directed against CaM KI and then stimulated with E2 as indicated in the "materials and methods". Phosphorylation of p53 at S366 was analyzed by western blot (N=4, \pm S.E.). b, siRNA to CaM KI 
blocks its expression in MCF-7 cells. MCF-7 cells were transfected as in panel a and analyzed by western blot for CaM KI or ERK (as a loading and transfection control) ( $\mathrm{N}=8, \pm$ S.E.). c, CaM KK is sufficient to stimulate p53 phosphorylation at S366. MCF-7 cells were transfected with control plasmid or plasmids containing constitutively active CaM KK and endogenous p53 phosphorylation was measured by western blotting ( $N=4, \pm$ S.E. $)$. d, siCaM KI inhibits carbachol-stimulation of p53 phosphorylation. MDA cells were transfected similar to panel a, treated with carbachol and p53 phosphorylation was measured by western blotting ( $\mathrm{N}=4, \pm \mathrm{S}$.E.). e, CaM KI protein is silenced in MDA cells. MDA cells were transfected with control scrambled siRNAs or siRNAs complementary to $\mathrm{CaM}$ KI and endogenous ERK and CaM KI were measured by western blotting as in panel $\mathbf{b}(\mathrm{N}=4, \pm \mathrm{S} . \mathrm{E}$.). $\mathbf{f}$, CaM KK expression increases p53 phosphorylation. MDA cells were transfected as in panel c and p53 phosphorylation was measured and analyzed as indicated $(\mathrm{N}=4, \pm$ S.E.).

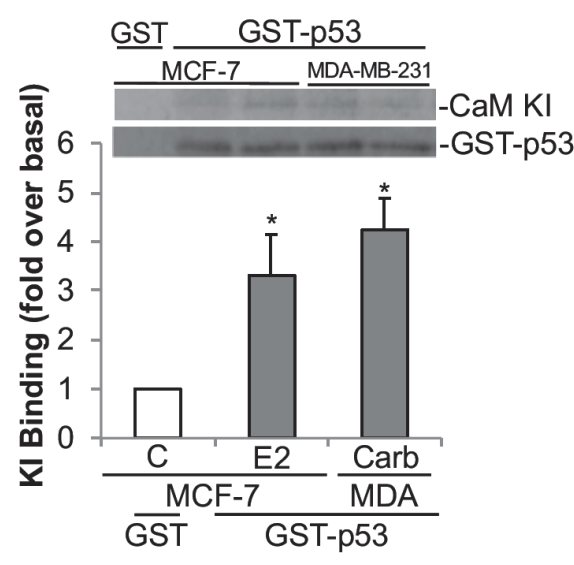

(a)

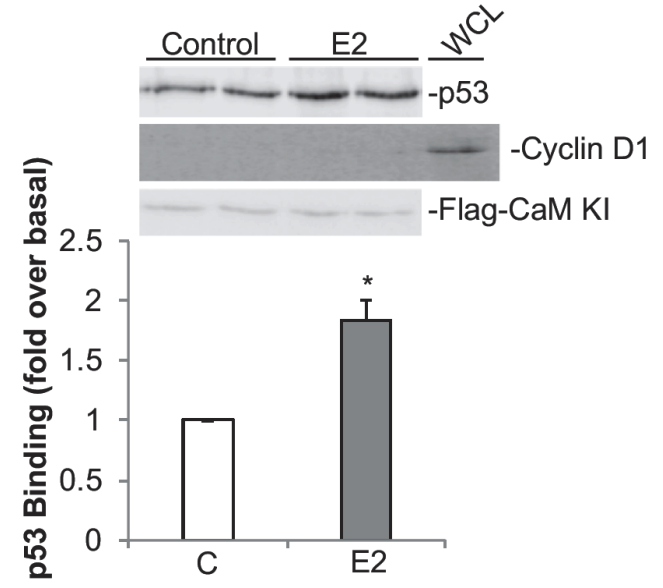

(b)

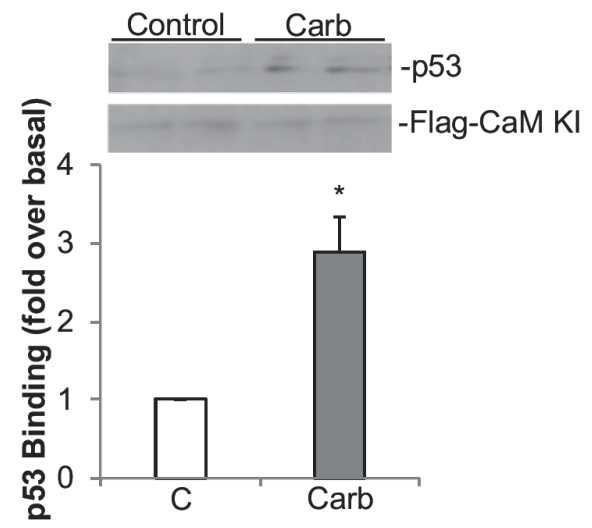

(c)

Fig. 4 CaM KI and p53 proteins associate. a, Purified p53 protein binds to CaM KI. MCF-7 cells received no pretreatment $(\mathrm{C})$ or were treated with $\mathrm{E} 2(10 \mathrm{nM})$ and MDA cells were treated with carbachol $(100 \mathrm{mM})$ as in previous figures. Endogenous proteins were prepared from cell lysates and incubated with GST-p53 as described in the "materials and methods". The presence of CaM KI from the indicated cell lysates that associated with GST-p53 was then analyzed by western blot $(\mathrm{N}=4, \pm$ S.E.). b, E2 enhances CaM KI and p53 binding. MCF-7 cells were transfected with Flag-CaM KI and then treated with E2 as indicated and Flag- CaM KI was immunoprecipitated and analyzed by western blot for Flag, Cyclin D1 or p53 (N=4, \pm S.E.). c, CaM KI and p53 associate in MDA cells. MDA cells were transfected with Flag-CaM KI then stimulated with carbachol or left untreated (C) and Flag-CaM KI was immunoprecipitated and p53 association was measured by western blotting ( $=4, \pm$ S.E.). 


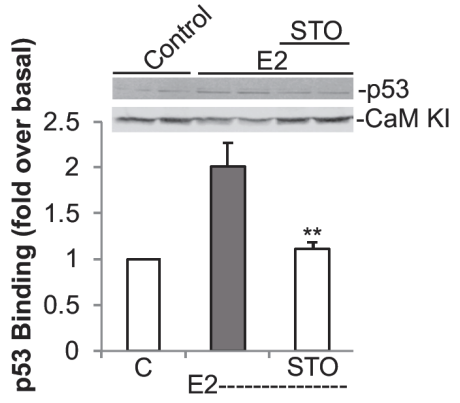

(a)

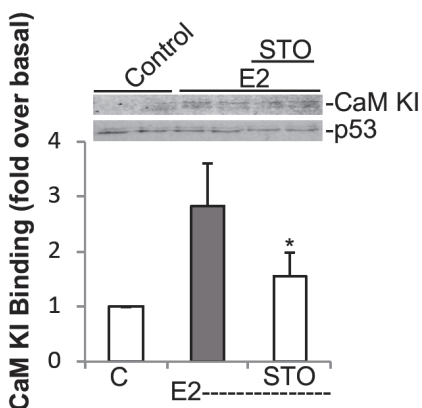

(b)

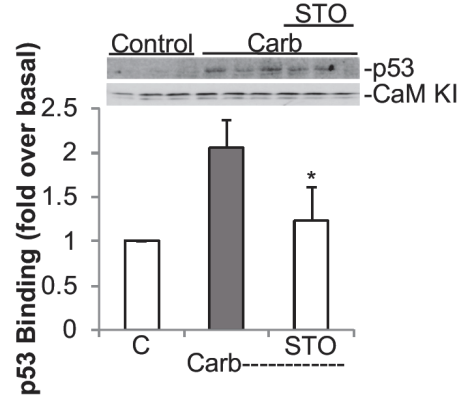

(c)

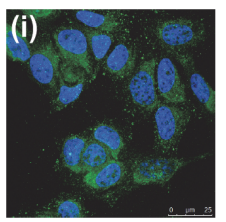

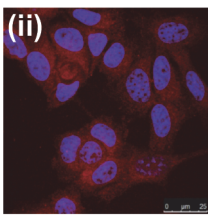

(d)
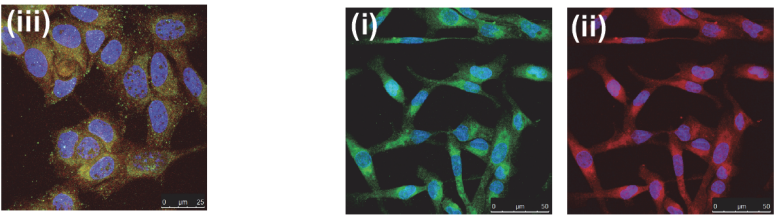

(e)

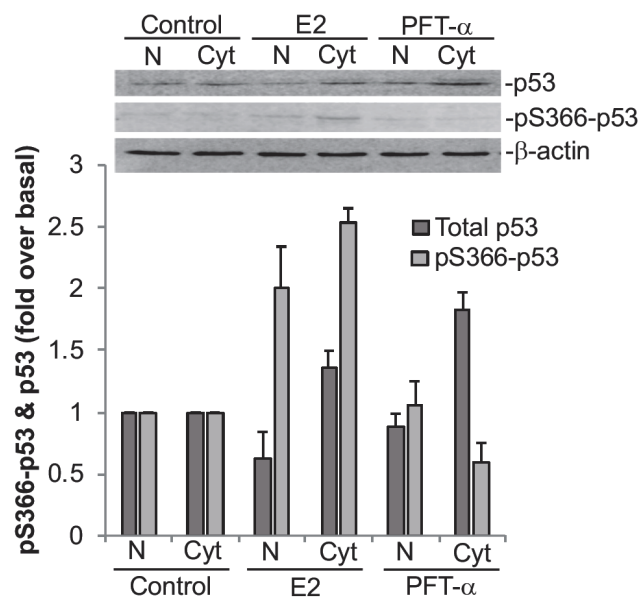

(f)

Fig. 5 p53 and CaM KI interact in breast cancer cells. a, E2 stimulates enhances endogenous CaM KI-p53

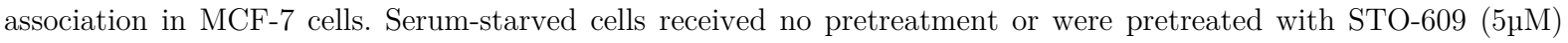
and then stimulated with E2 (10nM) for 5 minutes as indicated. CaM KI was immunoprecipitated and endogenous p53 binding was examined by western blot ( $\mathrm{N}=6, \pm$ S.E.). b. Endogenous p53 pulls-out CaM KI. MCF-7 cells were treated identical to panel a, and CaM KI binding was evaluated by western blot ( $\mathrm{N}=6, \pm$ S.E.). c, Endogenous CaM KI binds p53 in MDA cells. Cells were pretreated with STO-609 $(5 \mu \mathrm{M})$ and then stimulated with carbachol $(100 \mathrm{mM})$ as indicated. CaM KI was immunoprecipitated and extracts western blotted, and probed for p53 as done in previous panels ( $\mathrm{N}=6, \pm$ S.E.). d, CaM KI and p53 co-localize in MCF-7 cells. MCF-7 cells were stimulated with E2 $(10 \mathrm{nM})$ for 5 minutes and evaluated as described in the "materials and methods" using confocal microscopy and imaged using the 63X objective $(\mathrm{N}=4)$. $\mathbf{i}$, Endogenous CaM KI (green) is primarily cytoplasmic in MCF-7 cells and nuclei are blue. ii, p53 (red) is found in the cytoplasm and nucleus (blue) of MCF-7 cells. iii, p53 and CaM KI proteins co-localize in the cytoplasm (yellow/orange) of cells. e, Endogenous p53 and CaM KI proteins associate in MDA cells. MDA cells were stimulated with carbachol $(100 \mathrm{mM})$ for 5 minutes and evaluated using confocal microscopy and imaged using the 63X objective $(\mathrm{N}=4)$. $\mathbf{i}$, Active CaM KI (green) is found the in the cytoplasm of MDA cells, blue (nuclei). ii, p53 protein (red) localizes primarily in the cytoplasm of carbachol-treated MDA cells, the nucleus is stained blue. iii, Endogenous p53 and CaM KI co-localize (yellow/orange) in the cytoplasm and perinuclear region of MDA cells. Nuclei are shown in blue $(\mathrm{N}=4)$. f, Phosphorylated p53 is enriched in the cytoplasm of MCF-7 cells. MCF-7 cells were serum-starved and then treated with E2 (10nM) or PFT- $\alpha$ ( $5 \mu \mathrm{M})$ for 5 minutes as indicated. Lysates were then fractionated into either nuclear $(\mathrm{N})$ or cytoplasmic $(\mathrm{Cyt})$ components and analyzed by western blot as described in the methods $(\mathrm{N}=3)$. 


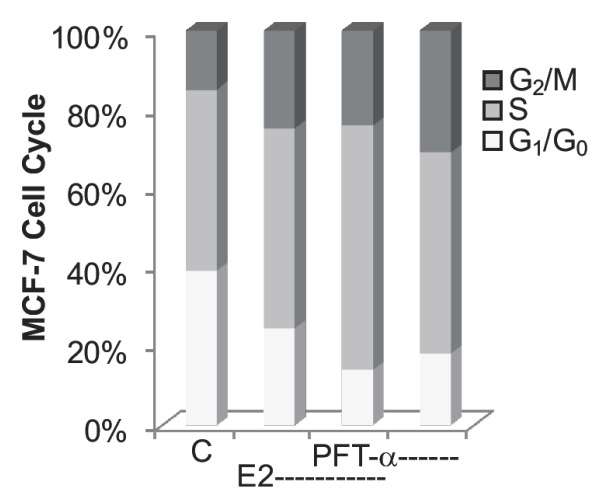

(a)

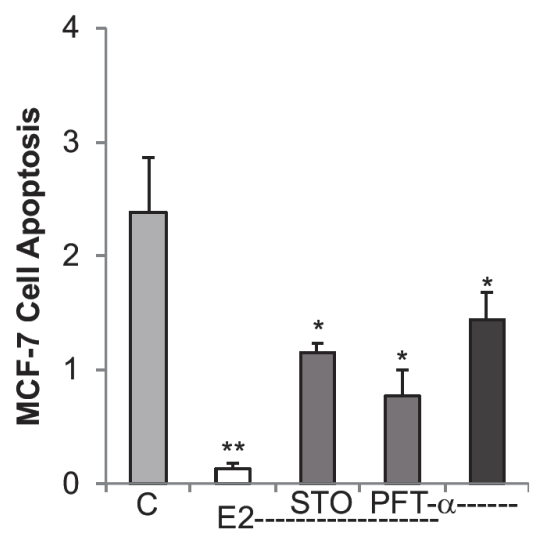

(c)

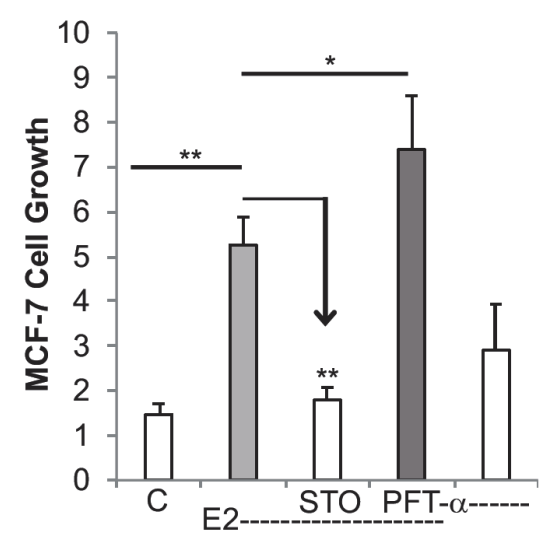

(b)

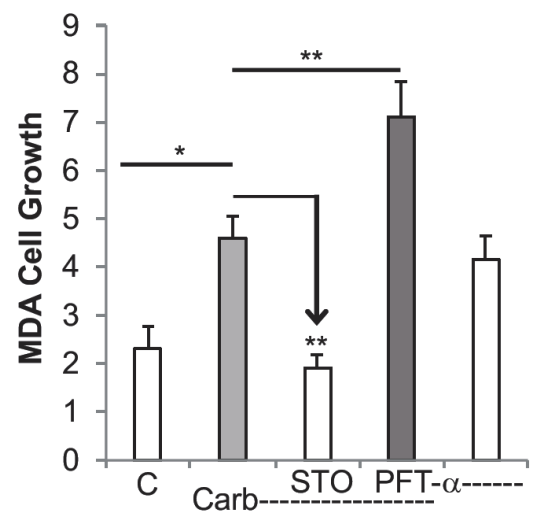

(d)

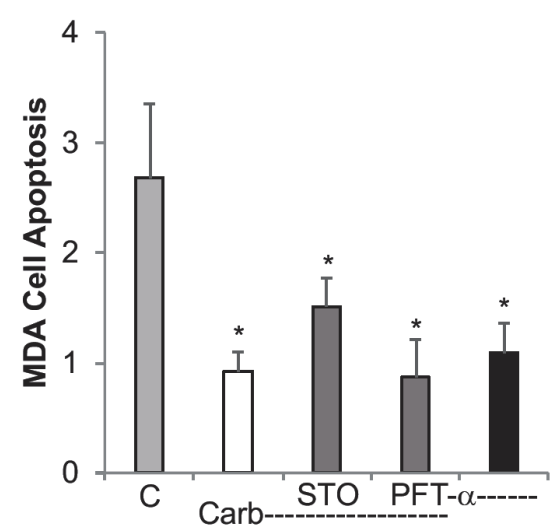

(e)

Fig. 6 p53 and CaM KI control breast cancer cell growth and apoptosis. a, E2 and PFT- $\alpha$ increase MCF7 cell cycle progression. Serum-starved cells were stimulated with E2 (10nM), PFT- $\alpha(5 \mu \mathrm{M})$ or the combination for 96 hours and the numbers of cells in each phase was analyzed $(\mathrm{N}=4)$. $\mathbf{b}$, E2 utilizes CaM KK and p53 to regulate

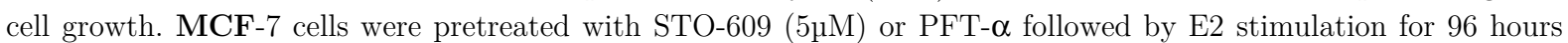
and the number of cells was quantified and analyzed. Comparisons for significance are indicated with numbers over lines or arrows and their corresponding histogram bars $(\mathrm{N}=4, \pm$ S.E.). c, CaM Kinase inhibition of p53 increases cell survival in MCF-7 cells. MCF-7 cells were treated identically to figure b and then measured and analyzed for apoptosis ( $\mathrm{N}=4, \pm$ S.E.). d, Carbachol enhances MDA cell proliferation through p53. MDA cells were serum-starved and treated with carbachol $(100 \mathrm{mM})$ or pretreated with STO-609 $(5 \mu \mathrm{M})$ or PFT- $\alpha(5 \mu \mathrm{M})$ and then stimulated with carbachol for 96 hours and cell growth was evaluated and analyzed. Comparisons for significance are indicated with numbers over lines or arrows and their corresponding histogram bars $(\mathrm{N}=4, \pm \mathrm{S}$.E.). e, CaM KI and p53 mediate 
carbachol's inhibition of apoptosis in MDAs. MDA cells were serum starved and treated the same as in figure d and then examined and analyzed for apoptosis at 96 hours $(\mathrm{N}=4, \pm$ S.E. $)$.

Acknowledgments. This work was supported in part through a grant from the M.J. Murdock Charitable Trust to J.M.S. \#2009261 and \#2011267. This work was also supported in part through the Jack S. Holman Endowment fund to J.M.S and the Paul K. and Evalyn E.C. Richter Memorial Fund at George Fox University.

Conflict of interest: The authors declare that they have no conflict of interest.

\section{References}

1. Brzozowski, J.S. and K.A. Skelding, The Multi-Functional Calcium/Calmodulin Stimulated Protein Kinase (CaMK) Family: Emerging Targets for Anti-Cancer Therapeutic Intervention. Pharmaceuticals (Basel), 2019. $12(1)$

2. Simon, B., A.S. Huart, and M. Wilmanns, Molecular mechanisms of protein kinase regulation by calcium/calmodulin. Bioorg Med Chem, 2015. 23(12): p. 2749-60.

3. Takemoto-Kimura, S., et al., Calmodulin kinases: essential regulators in health and disease. J Neurochem, 2017. 141(6): p. 808-818.

4. Bayer, K.U. and H. Schulman, CaM Kinase: Still Inspiring at 40. Neuron, 2019. 103(3): p. 380-394.

5. Schmitt, J.M., et al., Calcium activation of ERK mediated by calmodulin kinase I. J Biol Chem, 2004. 279(23): p. 24064-72.

6. Schmitt, J.M., et al., Calmodulin-dependent kinase kinase/calmodulin kinase I activity gates extracellularregulated kinase-dependent long-term potentiation. J Neurosci, 2005. 25(5): p. 1281-90.

7. Schmitt, J.M., et al., ERK activation and cell growth require CaM kinases in MCF-7 breast cancer cells. Mol Cell Biochem, 2010. 335(1-2): p. 155-71.

8. Kahl, C.R. and A.R. Means, Regulation of cyclin D1/Cdk4 complexes by calcium/calmodulin-dependent protein kinase I. J Biol Chem, 2004. 279(15): p. 15411-9.

9. Lu, F., et al., Downregulation of CREB Promotes Cell Proliferation by Mediating G1/S Phase Transition in Hodgkin Lymphoma. Oncol Res, 2016. 24(3): p. 171-9.

10. Sethi, S., et al., Social Context Enhances Hormonal Modulation of Pheromone Detection in Drosophila. Curr Biol, 2019. 29(22): p. 3887-3898 e4.

11. Mallampalli, R.K., et al., Fbxl12 triggers G1 arrest by mediating degradation of calmodulin kinase I. Cell Signal, 2013.

12. Kang, X., et al., CAMKs support development of acute myeloid leukemia. J Hematol Oncol, 2018. 11(1): p. 30.

13. Davare, M.A., T. Saneyoshi, and T.R. Soderling, Calmodulin-kinases regulate basal and estrogen stimulated medulloblastoma migration via Rac1. J Neurooncol, 2011. 104(1): p. 65-82.

14. Jadeja, R.N., et al., M3 muscarinic receptor activation reduces hepatocyte lipid accumulation via CaMKKbeta/AMPK pathway. Biochem Pharmacol, 2019. 169: p. 113613.

15. Penfold, L., et al., CAMKK2 Promotes Prostate Cancer Independently of AMPK via Increased Lipogenesis. Cancer Res, 2018. 78(24): p. 6747-6761.

16. Tokumitsu, H., et al., A single amino acid difference between alpha and beta Ca2+/calmodulin-dependent protein kinase kinase dictates sensitivity to the specific inhibitor, STO-609. J Biol Chem, 2003. 278(13): p. 10908-13.

17. Rodriguez-Mora, O.G., et al., Calcium/calmodulin-dependent kinase I and calcium/calmodulin-dependent kinase kinase participate in the control of cell cycle progression in MCF-7 human breast cancer cells. Cancer Res, 2005. 65(12): p. 5408-16.

18. Brufsky, A.M. and M.N. Dickler, Estrogen Receptor-Positive Breast Cancer: Exploiting Signaling Pathways Implicated in Endocrine Resistance. Oncologist, 2018. 23(5): p. 528-539.

19. Jameera Begam, A., S. Jubie, and M.J. Nanjan, Estrogen receptor agonists/antagonists in breast cancer therapy: A critical review. Bioorg Chem, 2017. 71: p. 257-274.

20. Improta-Brears, T., et al., Estrogen-induced activation of mitogen-activated protein kinase requires mobilization of intracellular calcium. Proc Natl Acad Sci U S A, 1999. 96(8): p. 4686-91. 
21. Pedram, A., et al., Developmental phenotype of a membrane only estrogen receptor alpha (MOER) mouse. J Biol Chem, 2009. 284(6): p. 3488-95.

22. Bertheau, P., et al., p53 in breast cancer subtypes and new insights into response to chemotherapy. Breast, 2013. 22 Suppl 2: p. S27-9.

23. Meek, D.W., Regulation of the p53 response and its relationship to cancer. Biochem J, 2015. 469(3): p. 325-46.

24. Chen, J., The Cell-Cycle Arrest and Apoptotic Functions of p53 in Tumor Initiation and Progression. Cold Spring Harb Perspect Med, 2016. 6(3): p. a026104.

25. Vousden, K.H. and C. Prives, Blinded by the Light: The Growing Complexity of p53. Cell, 2009. 137(3): p. 41331.

26. Qu, L., et al., Endoplasmic reticulum stress induces p53 cytoplasmic localization and prevents p53-dependent apoptosis by a pathway involving glycogen synthase kinase-3beta. Genes Dev, 2004. 18(3): p. 261-77.

27. Yogosawa, S. and K. Yoshida, Tumor suppressive role for kinases phosphorylating 553 in DNA damage-induced apoptosis. Cancer Sci, 2018. 109(11): p. 3376-3382.

28. Rajagopalan, S., et al., 14-3-3 activation of DNA binding of p53 by enhancing its association into tetramers. Nucleic Acids Res, 2008. 36(18): p. 5983-91.

29. Schmitt, J.M., et al., Estrogen Activation of CaM Kinases and Transcription Is Blocked by Vitamin D in MCF7 Breast Cancer Cells. Journal of Advances in Molecular Biology, 2017. 1(3): p. 129-147.

30. Schmitt, J.M., et al., CaM kinase control of AKT and LNCaP cell survival. J Cell Biochem, 2012. 113(5): p. 1514-26.

31. Gocher, A.M., et al., Akt activation by Ca(2+)/calmodulin-dependent protein kinase kinase 2 (CaMKK2) in ovarian cancer cells. J Biol Chem, 2017. 292(34): p. 14188-14204.

32. Zhang, X., M.R. Diaz, and D. Yee, Fulvestrant regulates epidermal growth factor (EGF) family ligands to activate EGF receptor (EGFR) signaling in breast cancer cells. 2013. 139(2): p. 351-360.

33. Chi, M., et al., Phosphorylation of calcium/calmodulin-stimulated protein kinase II at T286 enhances invasion and migration of human breast cancer cells. Sci Rep, 2016. 6: p. 33132.

34. Foster, J.S., et al., Estrogens down-regulate p27Kip1 in breast cancer cells through Skp2 and through nuclear export mediated by the ERK pathway. J Biol Chem, 2003. 278(42): p. 41355-66.

35. Fredersdorf, S., et al., High level expression of p27(kip1) and cyclin D1 in some human breast cancer cells: inverse correlation between the expression of p27(kip1) and degree of malignancy in human breast and colorectal cancers. Proc Natl Acad Sci U S A, 1997. 94(12): p. 6380-5.

36. Li, W., et al., A truncated p53 in human lung cancer cells as a critical determinant of proliferation and invasiveness. Tumor Biology, 2017. 39(6): p. 101042831770382.

37. Komarov, P.G., et al., A chemical inhibitor of 553 that protects mice from the side effects of cancer therapy. Science, 1999. 285(5434): p. 1733-7.

38. Li, Q., et al., Genetic analysis of p53 nuclear importation. Oncogene, 2007. 26(57): p. 7885-93.

39. Liang, S.H. and M.F. Clarke, A bipartite nuclear localization signal is required for p53 nuclear import regulated by a carboxyl-terminal domain. J Biol Chem, 1999. 274(46): p. 32699-703.

40. Liang, S.H. and M.F. Clarke, The nuclear import of 553 is determined by the presence of a basic domain and its relative position to the nuclear localization signal. Oncogene, 1999. 18(12): p. 2163-6.

41. Lin, F., et al., GTSE1 is involved in breast cancer progression in p53 mutation-dependent manner. Journal of Experimental \& Clinical Cancer Research, 2019. 38(1).

42. Korkmaz, G., et al., A CRISPR-Cas9 screen identifies essential CTCF anchor sites for estrogen receptor-driven breast cancer cell proliferation. Nucleic Acids Research, 2019. 47(18): p. 9557-9572.

43. Bailey, S.T., et al., Estrogen receptor prevents p53-dependent apoptosis in breast cancer. Proc Natl Acad Sci U S A, 2012. 109(44): p. 18060-5.

44. Ou, Y.H., et al., p53 C-terminal phosphorylation by CHK1 and CHK2 participates in the regulation of DNAdamage-induced C-terminal acetylation. Mol Biol Cell, 2005. 16(4): p. 1684-95.

45. Rajagopalan, S., et al., Mechanistic differences in the transcriptional activation of p53 by 14-3-3 isoforms. Nucleic Acids Res, 2010. 38(3): p. 893-906.

46. van Dieck, J., et al., Posttranslational modifications affect the interaction of S100 proteins with tumor suppressor p53. J Mol Biol, 2009. 394(5): p. 922-30.

47. Mueller, A., et al., The calcium-binding protein S100A2 interacts with p53 and modulates its transcriptional activity. J Biol Chem, 2005. 280(32): p. 29186-93. 